\title{
Correction to: Robust classification via MOM minimization
}

\author{
Guillaume Lecué $^{1}$ D $\cdot$ Matthieu Lerasle ${ }^{1} \cdot$ Timothée Mathieu $^{2}$
}

Published online: 3 June 2020

(c) The Author(s), under exclusive licence to Springer Science+Business Media LLC, part of Springer Nature 2020

\section{Correction to: Machine Learning https://doi.org/10.1007/s10994-019-05863-6}

There is a mistake in one of the authors' names (in both online and print versions of the article): it should be Timothée Mathieu instead of Timlothée Mathieu.

Publisher's Note Springer Nature remains neutral with regard to jurisdictional claims in published maps and institutional affiliations.

The original article can be found online at https://doi.org/10.1007/s10994-019-05863-6.

\footnotetext{
Guillaume Lecué

Guillaume.lecue@ensae.fr

Matthieu Lerasle

matthieu.lerasle@ensae.fr

Timothée Mathieu

timothee.mathieu@u-psud.fr

1 CREST-ENSAE, IPParis, Palaiseau, France

2 Université Paris Orsay, Orsay, France
} 